\title{
Observation of a threshold effect in the anomalous $\mathbf{J} / \psi$ suppression
}

\author{
M.C. Abreu ${ }^{7, a)}$, B. Alessandro ${ }^{12)}$, C. Alexa ${ }^{4)}$, R. Arnaldi ${ }^{12)}$, J. Astruc ${ }^{9)}$, M. Atayan ${ }^{14)}$, \\ C. Baglin ${ }^{2)}$, A. Baldit ${ }^{3)}$, M. Bedjidian ${ }^{13)}$, F. Bellaiche ${ }^{13)}$, S. Beolè̀ ${ }^{12)}$, V. Boldea ${ }^{4)}$, \\ P. Bordalo ${ }^{7, b)}$, A. Bussière ${ }^{2)}$, V. Capony ${ }^{2)}$, L. Casagrande ${ }^{7)}$, J. Castor $^{3)}$, T. Chambon ${ }^{3)}$, \\ B. Chaurand ${ }^{10)}$, I. Chevrot ${ }^{3)}$, B. Cheynis ${ }^{13)}$, E. Chiavassa ${ }^{12)}$, C. Cicalò ${ }^{5)}$, M.P. Comets ${ }^{9)}$, \\ N. Constans ${ }^{10)}$, S. Constantinescu ${ }^{4)}$, J. Cruz ${ }^{7)}$, A. De Falco ${ }^{5)}$, G. Dellacasa ${ }^{1)}$, \\ N. De Marco $^{12)}$, A. Devaux ${ }^{3)}$, S. Dita ${ }^{4)}$, O. Drapier ${ }^{13)}$, B. Espagnon ${ }^{3)}$, J. Fargeix ${ }^{3)}$, \\ S.N. Filippov ${ }^{8)}$, F. Fleuret ${ }^{10)}$, P. Force ${ }^{3)}$, M. Gallio ${ }^{12)}$, L. Gatignon ${ }^{6)}$, Y.K. Gavrilov ${ }^{8)}$, \\ C. Gerschel ${ }^{9)}$, P. Giubellino ${ }^{12)}$, M.B. Golubeva ${ }^{8)}$, M. Gonin ${ }^{10)}$, A.A. Grigorian ${ }^{14)}$, \\ J.Y. Grossiord ${ }^{13)}$, F.F. Guber ${ }^{8)}$, A. Guichard ${ }^{13)}$, H. Gulkanyan ${ }^{14)}$, R. Hakobyan ${ }^{14)}$, \\ R. Haroutunian ${ }^{13)}$, M. Idzik ${ }^{12, c)}$, D. Jouan ${ }^{9)}$, T.L. Karavitcheva ${ }^{8)}$, L. Kluberg ${ }^{10)}$, \\ A.B. Kurepin ${ }^{8)}$, Y. Le Bornec ${ }^{9)}$, C. Lourenço ${ }^{6)}$, L. Luquin ${ }^{3)}$, M. Mac Cormick ${ }^{9}$, \\ P. Macciotta ${ }^{5)}$, A. Marzari-Chiesa ${ }^{12)}$, M. Masera ${ }^{12)}$, A. Masoni ${ }^{5)}$, S. Mehrabyan ${ }^{14)}$, \\ M. Monteno ${ }^{12)}$, S. Mourgues ${ }^{3)}$, A. Musso ${ }^{12)}$, F. Ohlsson-Malek ${ }^{13, d)}$, P. Petiau ${ }^{10)}$, \\ A. Piccotti ${ }^{12)}$, J.R. Pizzi ${ }^{13)}$, W.L. Prado da Silva ${ }^{12, e)}$, F. Prino ${ }^{12)}$, G. Puddu ${ }^{5)}$, \\ C. Quintans ${ }^{7)}$, C. Racca ${ }^{11)}$, L. Ramello ${ }^{1)}$, S. Ramos ${ }^{7, b)}$, P. Rato-Mendes ${ }^{12)}$, L. Riccati ${ }^{12)}$, \\ A. Romana ${ }^{10)}$, I. Ropotar ${ }^{6)}$, P. Saturnini ${ }^{3)}$, E. Scomparin ${ }^{6, f)}$, S. Serci ${ }^{5)}$, R. Shahoyan ${ }^{7, g)}$, \\ S. Silva ${ }^{7)}$, M. Sitta ${ }^{1)}$, C. Soave ${ }^{12)}$, P. Sonderegger ${ }^{6, b)}$, X. Tarrago ${ }^{9)}$, P. Temnikov ${ }^{5)}$, \\ N.S. Topilskaya ${ }^{8)}$, G.L. Usai ${ }^{5)}$, E. Vercellin ${ }^{12)}$, N. Willis ${ }^{9)}$
}

\section{NA50 Collaboration}

\begin{abstract}
We report on a search for a phase transition from ordinary nuclear matter to a state of deconfined quarks and gluons as predicted by lattice QCD calculations. A new measurement of charmonium production in $\mathrm{Pb}-\mathrm{Pb}$ interactions at $158 \mathrm{GeV} / \mathrm{c}$ per nucleon agrees with our previous results and confirms the anomalous $\mathrm{J} / \psi$ suppression we had already observed on a significantly smaller data sample. New event selection and analysis techniques show that, for peripheral collisions, the $\mathrm{J} / \psi$ cross-section per nucleon-nucleon collision agrees with the precise suppression pattern inferred from a wide range of measurements extending from p-p up to S-U collisions. As the collisions become more central, the $\mathrm{Pb}-\mathrm{Pb}$ cross-section exhibits a clear departure from this normal behaviour. The onset of the anomalous $\mathrm{J} / \psi$ suppression reported here is the first clear observation of a threshold effect in heavy ion collisions and can be considered as a strong indication of the production of a deconfined quark-gluon phase in central $\mathrm{Pb}-\mathrm{Pb}$ collisions.
\end{abstract}


1) Università del Piemonte Orientale, Alessandria and INFN-Torino, Italy

2) Laboratoire de Physique des Particules (LAPP), IN2P3-CNRS, Annecy-le-Vieux, France

3) Laboratoire de Physique Corpusculaire (LPC), Université Blaise Pascal, IN2P3-CNRS, Aubière, France

4) Institute of Atomic Physics (IFA), Bucharest, Romania

5) Università di Cagliari/INFN, Cagliari, Italy

6) CERN, Geneva, Switzerland

7) Laboratório de Instrumentação e Física Experimental de Partículas (LIP), Lisbon, Portugal

8) Institute for Nuclear Research (INR), Moscow, Russia

9) Institut de Physique Nucléaire de Orsay (IPNO), Université Paris-Sud, IN2P3-CNRS, Orsay, France

10) Laboratoire de Physique Nucléaire des Hautes Energies (LPNHE), Ecole

Polytechnique, IN2P3-CNRS, Palaiseau, France

11) Centre de Recherches Nucléaires, Université Louis Pasteur, IN2P3-CNRS, Strasbourg, France

12) Università di Torino/INFN, Torino, Italy

13) Institut de Physique Nucléaire de Lyon (IPNL), Université Claude Bernard, IN2P3-CNRS, Villeurbanne, France

14) Yerevan Physics Institute (YerPhI), Yerevan, Armenia

a) Also at UCEH, Universidade do Algarve, Faro, Portugal

b) Also at IST, Universidade Técnica de Lisboa, Lisbon, Portugal

c) Now at FPNT, University of Mining and Metallurgy, Cracow, Poland

d) Now at ISN, Univ. Joseph Fourier and CNRS-IN2P3, Grenoble, France

e) Now at UERJ, Rio de Janeiro, Brazil

f) On leave of absence from Università di Torino/INFN, Torino, Italy

g) On leave of absence from YerPhI, Yerevan, Armenia 


\section{Introduction}

In ordinary nuclear matter, quarks and gluons are confined within nucleons. Above the deconfinement temperature, $T_{c}$, as predicted by non-perturbative Quantum Chromodynamics (QCD) calculations, quarks and gluons are expected to behave as free objects within a new state of deconfined matter, the Quark-Gluon Plasma (QGP). It has been suggested that the phase transition between ordinary and deconfined matter could occur in ultrarelativistic heavy ion collisions where, under certain conditions, temperature and energy density could reach the required values. Heavy ion physics offers thus a unique opportunity to test a major prediction of QCD.

The suppression of charmonium states has been predicted to be an unambiguous signature of deconfinement [1]. The formation of the $c \bar{c}$ bound states would be suppressed in the QGP due to the screening of the attractive color force which binds the two quarks together. Charmonia are produced in the very early stage of the collision. Strongly bound states, like the $\mathrm{J} / \psi$, are expected to interact weakly with other hadrons. As a result, and more than other signatures, $\mathrm{J} / \psi$ suppression carries its original message through the different stages of the reacting medium.

Based on an extensive set of experimental data obtained with proton, oxygen and sulphur beams on several targets, the NA38 and NA51 experiments have indeed observed a $\mathrm{J} / \psi$ suppression which increases continuously and monotonically from the lighter to the heavier interacting nuclei [2]. The same behaviour is observed in sulphur-uranium collisions as a function of the centrality of the reaction. The observed suppression exhibits a completely smooth increasing pattern which is fully consistent with ordinary nuclear absorption of the charmonium states by the nucleons of the interacting nuclei. Within the framework of the Glauber model and from a simple first order exponential fit, the data lead to a $\mathrm{J} / \psi$ absorption cross-section of $6.1 \pm 0.7 \mathrm{mb}[3]$ which accounts for the observed suppression in a range which extends from p-p up to central S-U reactions.

The $\mathrm{J} / \psi$ suppression measured by experiment $\mathrm{NA} 50$ for $\mathrm{Pb}-\mathrm{Pb}$ interactions shows a clear departure from the smooth trend exhibited by measurements with lighter projectiles [3]. The data which led to the discovery of the anomalous $\mathrm{J} / \psi$ suppression were collected in 1995, at the CERN SPS. The $\mathrm{J} / \psi$ cross-section measured in $\mathrm{Pb}-\mathrm{Pb}$ at $158 \mathrm{GeV} / c$ per nucleon is a factor $0.71 \pm 0.03$ below the value extrapolated from the NA38/NA51 data according to pure nuclear absorption. Moreover, the observed anomaly increases with increasing centrality. Within a large statistical error, the results obtained for the most peripheral collisions have been found to be compatible with normal nuclear absorption as extrapolated from p-A and $\mathrm{S}-\mathrm{U}$ reactions.

In this paper we report the results from a high statistics sample of $\mathrm{Pb}-\mathrm{Pb}$ interactions, collected in 1996. The aim of this larger set of data is to increase significantly the sample of high mass muon pair events, in particular those produced in peripheral reactions. The new analysis presented hereafter is devoted to the study of $\mathrm{J} / \psi$ suppression as a function of centrality. It is intended to explore in detail the pattern of the observed anomaly and to compare, as accurately as possible, the most peripheral 
$\mathrm{Pb}-\mathrm{Pb}$ results with the previously studied $\mathrm{S}-\mathrm{U}$ reactions. Absolute cross-sections can be found in Ref. [4].

\section{$2 \quad$ Experimental setup}

The experimental setup used in 1996 was almost identical to the one used in 1995. The detector has been described in detail in Ref. [4]. Muon pairs are deflected by an air gap toroidal magnet in the pseudo-rapidity interval $2.8<\eta_{\text {lab }}<4.0$ and tracked by a set of multi-wire proportional chambers. A very forward ("zero degree" or ZDC) hadronic calorimeter [5] covers rapidities higher than 6.3 and measures essentially the energy, $E_{\mathrm{ZDC}}$, of the spectator (non-interacting) nucleons from the incoming $\mathrm{Pb}$ projectile.

The centrality of the collision is estimated by an electromagnetic calorimeter which measures $E_{\mathrm{T}}$, the neutral transverse energy produced in the reaction in the range $1.1<\eta_{\text {lab }}<2.3$. It should be underlined that this measurement suffers from a significant global absolute scale uncertainty as compared with similar measurements done in experiment NA38 in which a more central rapidity region was covered. We have thus renormalized the $E_{\mathrm{T}}$ absolute scale published in Ref. [4] or recently presented as preliminary results from the present data [6]. The new scale provides identical values for the 1995 and $1996 \mathrm{~Pb}$ data and is, furthermore, consistent with the NA38 $\mathrm{S}-\mathrm{U}$ results according to simulations done using the RQMD monte-carlo event generator [7]. The average correction multiplicative factor, already applied here, amounts to 0.81 for the 1996 preliminary results and to 0.74 for the 1995 published data. This change of $E_{\mathrm{T}}$ scale does not affect any other centrality estimator currently used in the analysis. The typical resolution achieved in the $E_{\mathrm{T}}$ measurement is $\sim 10 \%$ for $E_{\mathrm{T}}=40 \mathrm{GeV}$ and $\sim 7 \%$ for $E_{\mathrm{T}}=100 \mathrm{GeV}$.

The average beam intensity during the 1996 run was $5 \times 10^{7} \mathrm{~Pb}$ ions per burst as compared to $3 \times 10^{7}$ in 1995 . With a spill of $4.5 \mathrm{~s}$ nominal duration, the resulting pileup of two or more incident ions arriving within the $20 \mathrm{~ns}$ gate of the multichannel beam hodoscope amounted to $28 \%$ of the total sample of events. These pile-up events were tagged by the beam hodoscope and rejected from the final set of data. We recorded $\sim 2000$ triggers per burst. Besides the dimuon triggers, these included $10 \%$ of downscaled minimum bias triggers obtained by requiring only a non-zero energy deposit in the ZDC. A total of 170 million triggers (60 million in 1995) were written on tape. The target assembly was made of 7 sub-targets spaced $25 \mathrm{~mm}$ apart. Its total thickness was $12 \mathrm{~mm}$, a major change with respect to the 1995 setup which used $7 \mathrm{~mm}$ instead (i.e. $30 \%$ of an interaction length in 1996 against $17 \%$ in 1995). A major improvement with respect to 1995 was the better beam optics and sharply limited beam spot which resulted from a significantly higher primary beam intensity coupled to a severe upstream collimation. The fine tuning of the beam line led to a significant reduction of off-target background triggers with respect to the 1995 running conditions. The use of pre-interaction and halo detectors to tag 
beam interactions upstream from our target allowed, furthermore, a clean selection of on-target events. Finally, the contamination due to off-target dimuon events which survived the selection criteria could be estimated from the empty target special runs recorded during normal data taking. It amounts to about $3 \%$ of the events with $E_{\mathrm{T}}$ below $50 \mathrm{GeV}$ and is negligible for higher $E_{\mathrm{T}}$ values.

The final analysis of the 1996 run presented here is based on $190000 \mathrm{~J} / \psi$ events and 2100 Drell-Yan muon pairs with invariant mass higher than $4.2 \mathrm{GeV} / c^{2}$. This sample corresponds to an average fourfold increase in statistics as compared to the 1995 sample; as explained below and thanks to a new event selection procedure, the increase is significantly higher for peripheral collisions.

\section{The "standard" analysis}

The "standard" analysis method has already been described in Ref. [4]. The J/ $\psi$ and $\psi^{\prime}$ resonances are identified through their decays into muon pairs. The numbers of $\mathrm{J} / \psi$ and $\psi^{\prime}$ events and the number of Drell-Yan dimuons are obtained from the analysis of the muon pair invariant mass spectrum, taking into account the contributions originating from both the open charm associated production and the combinatorial background due to decays. The measured quantity is $B_{\mu \mu} \sigma_{\mathrm{J} / \psi} / \sigma_{\mathrm{DY}}$, the ratio of $\mathrm{J} / \psi$ to Drell-Yan cross-sections multiplied by the branching ratio of $\mathrm{J} / \psi$ into two muons. The branching ratio $B_{\mu \mu}$ is always included but generally omitted in the notations of this article. This quantity is referred to as the " $\mathrm{J} / \psi$ cross-section per nucleonnucleon collision" since the Drell-Yan cross-section is expected to be proportional to the number of elementary collisions in the reaction [3]. The study is done as a function of the neutral transverse energy $E_{\mathrm{T}}$ measured on an event by event basis and used as an estimator of the centrality.

\subsection{Data selection}

The muon pairs selected for the final data analysis have to satisfy the normal selection criteria already detailed in Ref. [4]. This selection has been modified for the analysis of the 1996 data as detailed hereafter.

The target algorithm makes use of the signals given by the two quartz blades located off the beam axis, on the left and right sides of each sub-target, which measure a fraction of the particles emitted in the interaction [8]. It identifies the sub-target where the primary interaction took place. Furthermore, it can also identify, if any, interactions of the spectator fragments with downstream sub-targets, a type of events called "re-interactions". The identification of the interaction sub-target rejects efficiently most of the off-target events. Nevertheless, this powerful cleaning tool induces a significant loss for the most peripheral (low multiplicity) on-target reactions and fails to identify part of the re-interactions which, usually, are made of at least one very peripheral reaction. These two types of events give signals which are indistinguishable from those generated by the $\delta$ rays of the non interacting incident ions across 
the $\mathrm{Pb}$ targets. In order to recover the peripheral reactions, a new selection method has been developed which rejects most of the off-target contamination without requesting necessarily a positive response from the target algorithm (which is always used if present). The new method is based on the correlation between $E_{\mathrm{T}}$ and $E_{\mathrm{ZDC}}$ measured on an event by event basis by the two NA50 calorimeters. A contour-cut on the $E_{\mathrm{T}}-E_{\mathrm{ZDC}}$ correlation is applied, limited to the $2 \sigma$ distance from the average correlation. It efficiently rejects events originating both upstream and downstream from the target region and is therefore practically equivalent to a selection of $\mathrm{Pb}-\mathrm{Pb}$ events, as shown by the analysis of the empty target runs.
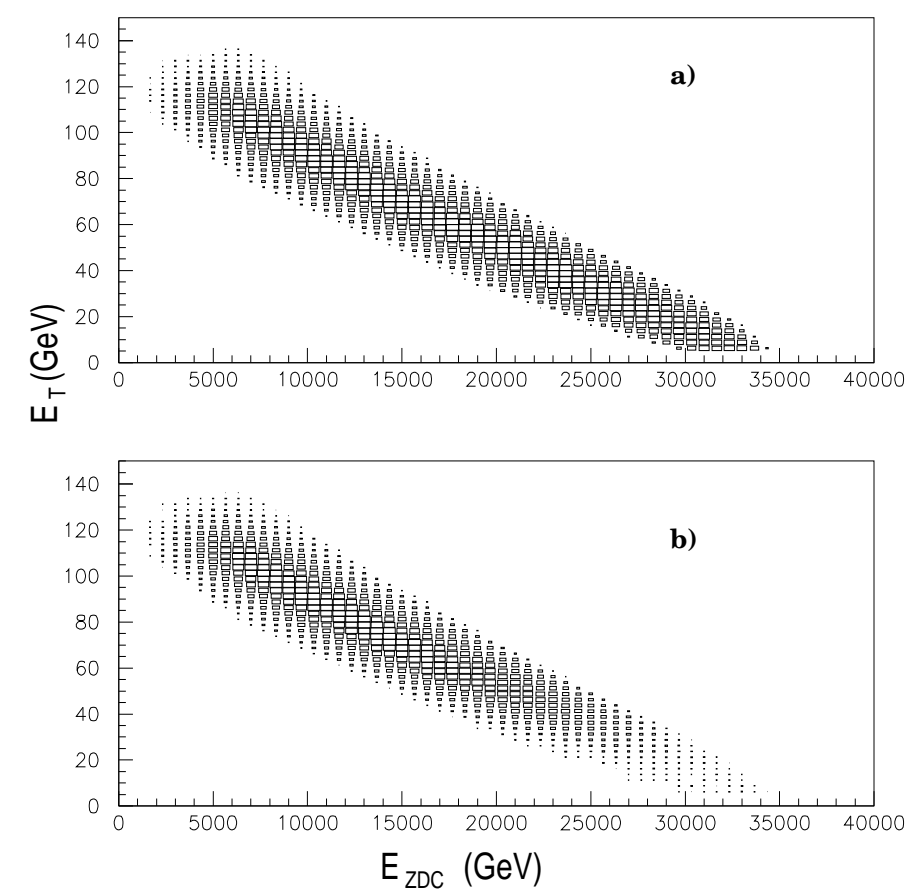

Figure 1: Correlation between neutral transverse energy and very forward hadronic energy for $\mathrm{J} / \psi$ events using the new selection procedure with (b) and without (a) requiring the vertex identification by the target algorithm.

Figure 1 shows the $E_{\mathrm{T}}-E_{\mathrm{ZDC}}$ correlation for high mass dimuons identified with the new method (a) and with the target algorithm (b). As can be seen, the new method recovers the sample of peripheral collisions rejected by the target algorithm. The contour-cut rejection applies to all events. The interaction sub-target is taken according to the response of the target algorithm, if any, and is arbitrarily considered to be the central (fourth) sub-target when the response is missing. In this last case, the resulting vertex smearing leads to a slightly worse resolution in the transverse energy and in the dimuon invariant mass calculations, since both depend on the precise interaction vertex. The mass resolution at the $\mathrm{J} / \psi$ peak, therefore, increases smoothly from 97 to $105 \mathrm{MeV}(3.1 \%$ to $3.4 \%)$ between central or semi-central and the most peripheral collisions. This variation is due to the increasing mass smearing 


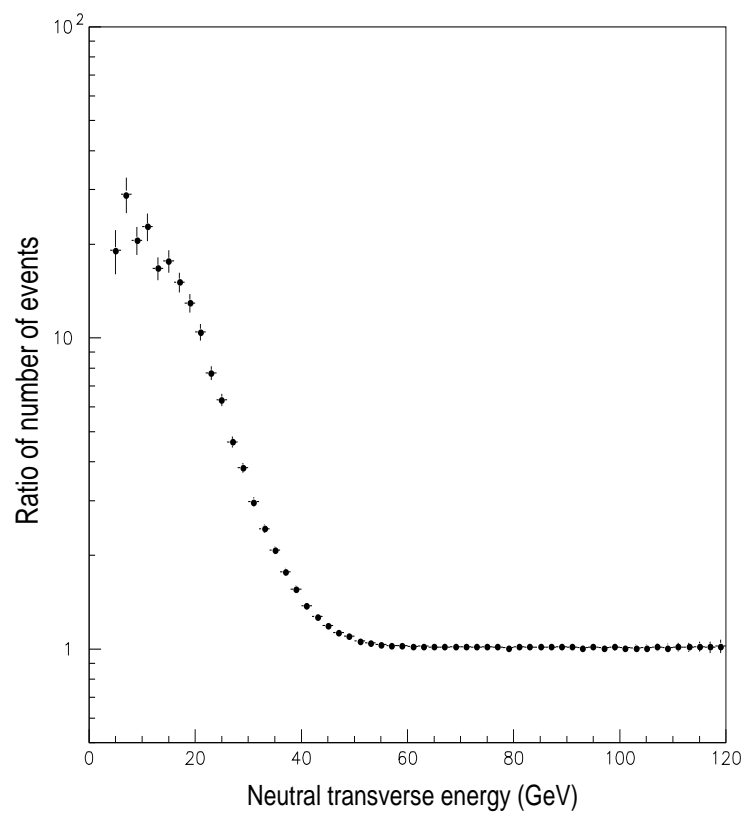

Figure 2: Ratio of the number of events selected by the contour-cut method and by the target algorithm.

and is well reproduced by Monte-Carlo simulations.

Figure 2 shows the ratio of the number of events selected by the contour-cut method and by the target algorithm. No event is recovered for $E_{\mathrm{T}}$ higher than $55 \mathrm{GeV}$ as, in this range, the target algorithm efficiency is close to unity, while for lower $E_{\mathrm{T}}$ values the number of events recovered by the contour-cut method increases smoothly as the collisions become more peripheral.

In order to reject dimuon events produced up-stream and down-stream from the target in $\mathrm{Pb}$-air interactions, severe cuts on the distance between the muon tracks and the beam axis are applied. The study of the $\psi^{\prime}$ dimuon vertex distribution along the beam axis, measured from the extrapolated muon tracks detected in the spectrometer, shows that these dimuon "vertex cuts" reduce the contribution of off-target events by a factor 3 .

Events identified by the target algorithm as re-interactions are rejected from the data analysis. They amount to $3 \%$ of the 1996 final analysis data sample (corresponding to $5.4 \%$ at the raw-data level), to be compared with $2 \%$ in the 1995 data. As explained above, the rate of reinteraction identification is lower than expected. For example, a target thickness corresponding to $30 \%$ of an interaction length should lead to a re-interaction contamination of 10-15\% (at the raw-data level). The reinteraction rejection inefficiency could induce a bias in the data analysis, which could be significant for values of $E_{\mathrm{T}}$ above $90 \mathrm{GeV}$, as suggested by the $E_{\mathrm{T}}$ distribution of the events positively tagged for re-interaction. 


\subsection{Analysis}

The final invariant mass spectrum of the opposite-sign pairs obtained from the whole sample of events collected in 1996 is shown in Fig. 3.

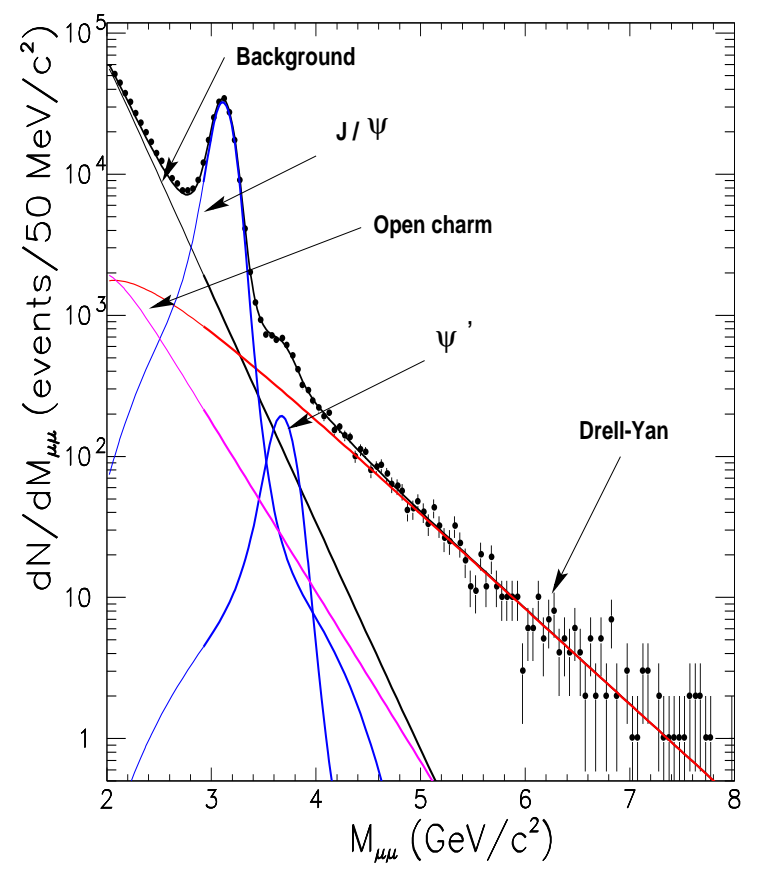

Figure 3: Opposite-sign muon pair invariant mass spectrum for $\mathrm{Pb}-\mathrm{Pb}$ collisions at $158 \mathrm{GeV} / c$ incident momentum.

It is fitted to the sum of five contributions, i.e. the combinatorial background, the open charm, $\mathrm{J} / \psi, \psi^{\prime}$ and Drell-Yan. The combinatorial background originating from uncorrelated $\pi$ and $\mathrm{K}$ decays is determined with the standard procedure using the sample of like-sign pairs. The shapes of the open charm, $\mathrm{J} / \psi, \psi^{\prime}$ and Drell-Yan contributions shown in Fig. 3 are determined by Monte-Carlo simulations. For open charm and Drell-Yan production, the simulation is based on PYTHIA [9] calculations using the MRS A [10] set of parton distribution functions. It has been checked that the results reported in this paper are insensitive to the specific set of parton distribution functions adopted for the analysis. The open charm continuum shown on the figure corresponds to $D \bar{D}$ semileptonic decays; its normalization is obtained from a fit to the intermediate mass region $2.2<M<2.9 \mathrm{GeV} / c^{2}$ with the Drell-Yan amplitude determined from the high mass part of the distribution.

The final fit is performed for $M \geq 2.9 \mathrm{GeV} / c^{2}$ with three free normalization parameters, i.e., the number of Drell-Yan, $\mathrm{J} / \psi$ and $\psi^{\prime}$ events. Additionally, the $\mathrm{J} / \psi$ mass is taken as a free parameter for better fit quality. The fitted value for the mass of the $\mathrm{J} / \psi$ is found to be systematically $0.3 \%$ higher than the value of the Particle Data Group [11], due to the average energy loss correction. The best fit gives a $\chi^{2} /$ dof $=1.03$ using the maximum likelihood method. The two-step fitting 
procedure is used to estimate the contribution of the $D \bar{D}$ "excess" [12] at high mass; it reduces to a negligible level the systematic error from this source on the $\sigma_{\mathrm{J} / \psi} / \sigma_{\mathrm{DY}}$ ratios. The global fit reproduces the trend of the data even below $2.9 \mathrm{GeV} / c^{2}$ as can be seen from Fig. 3, which suggests a clean selection and a fair agreement with the shapes of the different contributions.

\subsection{Results}

The ratios of cross-sections $\sigma_{\mathrm{J} / \psi} / \sigma_{\mathrm{DY}}$ are given integrating the Drell-Yan differential cross-section in the mass interval $2.9 \leq M \leq 4.5 \mathrm{GeV} / c^{2}$. The ratio $\sigma_{\mathrm{J} / \psi} / \sigma_{\mathrm{DY}}$ has been measured for the whole sample of $\mathrm{Pb}-\mathrm{Pb}$ events. From the 1996 data we obtain

$$
B_{\mu \mu} \sigma_{\mathrm{J} / \psi} / \sigma_{\mathrm{DY}}=17.0 \pm 0.2
$$

while from the 1995 data [4] we had obtained $16.1 \pm 0.4 \pm 0.2^{1}$.

The new ratio together with the now final analysis of the NA38/NA51 data [2] leads to

$$
R=0.77 \pm 0.04
$$

for the anomalous suppression factor as defined in Ref. [3] (ordinary nuclear absorption would correspond to $R=1$ ). This value is compatible with the value $0.71 \pm 0.03$ published in Ref. [3].

The ratio of the $\mathrm{J} / \psi$ to Drell-Yan cross-sections can be studied as a function of centrality. The analysis method described above is now applied in different $E_{\mathrm{T}}$ bins. For comparison purposes only, Fig. 4 shows the results obtained from the 1996 data with the coarse binning required by the limited number of events collected in 1995 .

The agreement between the two different samples is fairly good except for the highest $E_{\mathrm{T}}$ bin, which is attributed to a bias due to re-interaction type events (see section 3.1). Their mis-identification induces a smooth event migration from medium to high $E_{\mathrm{T}}$ values. It leads to an overestimated ratio of cross-sections for the upper end of the $E_{\mathrm{T}}$ range. This assumption is supported by the fact that re-interactions are expected to play a larger role for the 1996 data due to a significantly thicker Pb target. Further studies are therefore in progress to clarify the results for $E_{\mathrm{T}}>100 \mathrm{GeV}$.

The size of the 1996 data sample allows a more detailed centrality analysis in 15 different $E_{\mathrm{T}}$ bins. The results obtained for the ratios of $\mathrm{J} / \psi$ to Drell-Yan crosssections are listed in Table 1 together with the $\chi^{2} /$ dof of each individual fit. The quoted errors are purely statistical.

Figure 5 shows the cross-section ratios for the $15 E_{\mathrm{T}}$ bins presented in Table 1 (closed circles). In order to check the stability of the results, the figure shows also the cross-section ratios for bins shifted by half a bin (open circles). The curve represents the ordinary nuclear absorption fitted to the NA51 and NA38 results obtained with lighter projectiles [3]. It should be noted that, for the first time, the anomalous suppression pattern can be seen within the same data sample. It is clear from the

\footnotetext{
${ }^{1}$ The statistical error 0.3 published in Ref. [4] was underestimated.
} 


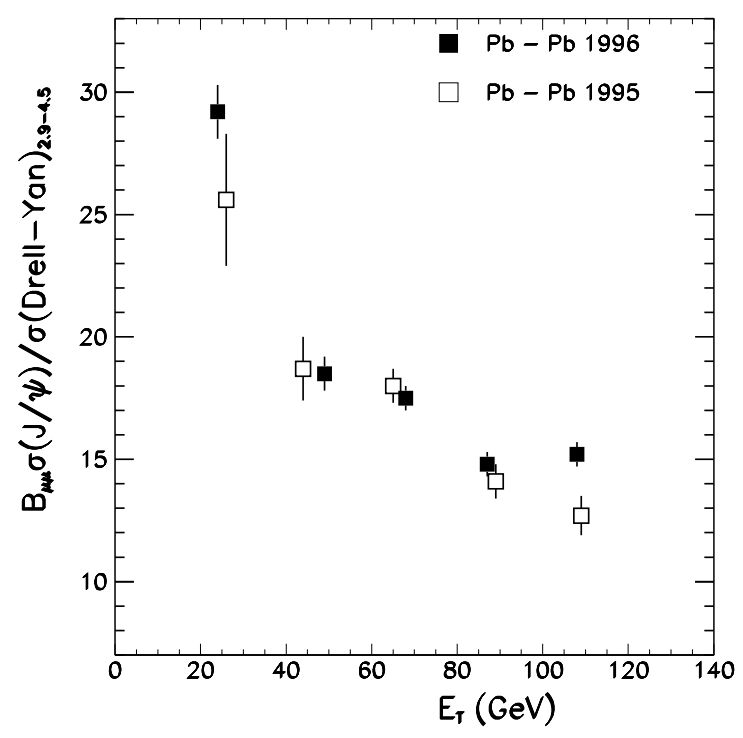

Figure 4: The standard $\sigma_{\mathrm{J} / \psi} / \sigma_{\mathrm{DY}}$ ratios for the 1995 and the 1996 data samples. The difference at the upper end of the $E_{\mathrm{T}}$ range is probably due to re-interactions in the target. See the text for details.

Table 1: Cross-section ratios for the $1996 \mathrm{~Pb}-\mathrm{Pb}$ data as a function of centrality. The ratios are given for Drell-Yan events in the mass interval $2.9-4.5 \mathrm{GeV} / c^{2}$. The values of $\left\langle E_{\mathrm{T}}\right\rangle$ and $\langle b\rangle$ correspond to the average neutral transverse energy and impact parameter for the events observed in each interval.

\begin{tabular}{rrrr}
\hline $\begin{array}{c}\left\langle E_{\mathrm{T}}\right\rangle \\
(\mathrm{GeV})\end{array}$ & $\begin{array}{c}\langle b\rangle \\
(\mathrm{fm})\end{array}$ & $B_{\mu \mu} \sigma_{\mathrm{J} / \psi} / \sigma_{\mathrm{DY}}$ & $\chi^{2} /$ dof \\
\hline 14 & 10.8 & $35.8 \pm 2.6$ & 1.08 \\
24 & 9.6 & $28.2 \pm 2.2$ & 1.20 \\
32 & 8.9 & $26.0 \pm 1.7$ & 0.84 \\
41 & 8.1 & $24.2 \pm 1.4$ & 0.85 \\
49 & 7.3 & $18.6 \pm 1.0$ & 1.22 \\
56 & 6.6 & $18.0 \pm 1.0$ & 1.02 \\
63 & 5.9 & $16.7 \pm 1.0$ & 1.24 \\
70 & 5.3 & $18.0 \pm 1.0$ & 1.32 \\
77 & 4.7 & $16.7 \pm 1.0$ & 0.83 \\
83 & 4.2 & $13.5 \pm 1.1$ & 0.90 \\
90 & 3.6 & $14.4 \pm 0.9$ & 0.94 \\
97 & 2.9 & $16.1 \pm 1.1$ & 1.20 \\
103 & 2.2 & $16.6 \pm 1.3$ & 0.98 \\
110 & 1.7 & $14.1 \pm 1.1$ & 0.88 \\
119 & 1.3 & $13.7 \pm 1.3$ & 0.52 \\
\hline
\end{tabular}




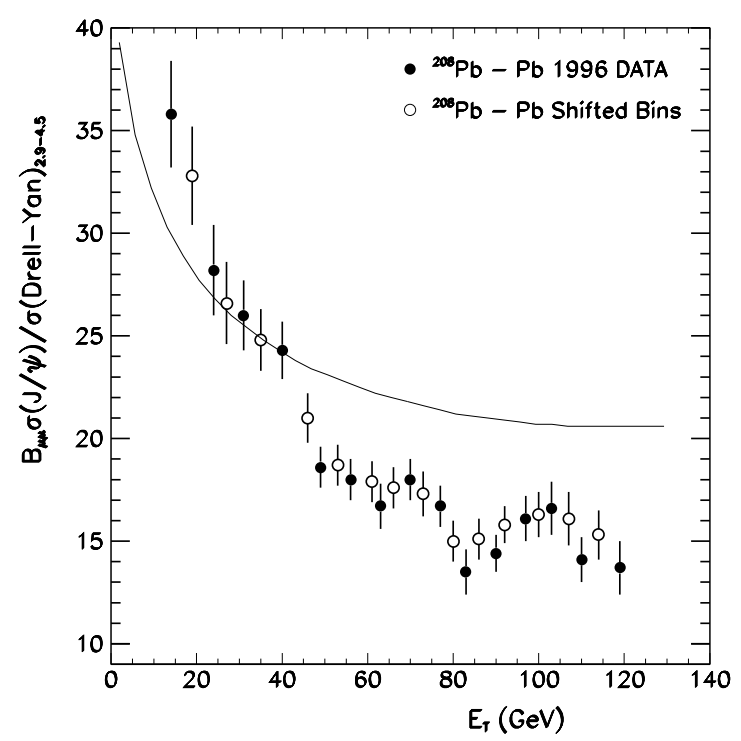

Figure 5: Ratios of $\mathrm{J} / \psi$ to Drell-Yan cross-sections as a function of centrality in $\mathrm{Pb}-\mathrm{Pb}$ collisions at $158 \mathrm{GeV} / \mathrm{c}$. The line corresponds to the fit to lighter interacting nuclei shown in Fig. 6. It is supposed to account for ordinary nuclear absorption (see text) with $\sigma_{\text {abs }}=6.4 \mathrm{mb}$.

figure that whereas for $E_{\mathrm{T}}<40 \mathrm{GeV} \mathrm{J} / \psi$ suppression agrees with measurements obtained from lighter systems, there is a significant departure from this behaviour for $E_{\mathrm{T}}>50 \mathrm{GeV}$. The onset of the anomaly can be seen as a drop of yield in a limited $10 \mathrm{GeV}$ wide $E_{\mathrm{T}}$ range which corresponds to an impact parameter $b \simeq 8 \mathrm{fm}$.

The ratio of the $\mathrm{J} / \psi$ to Drell-Yan cross-sections is plotted in Fig. 6 as a function of $L$ [13], the mean path length of the prompt $(c \bar{c})$ object through nuclear matter, which is related to $E_{\mathrm{T}}$ as explained in detail in Ref. [3]. The final results from the NA38 (p-W, p-U and S-U) and NA51 (p-p and p-d) experiments [2] are also presented in the figure. The NA38/NA51 measurements at $200 \mathrm{GeV} / c$ and $450 \mathrm{GeV} / c$ have been rescaled to $158 \mathrm{GeV} / c$ and corrected, together with the $\mathrm{Pb}-\mathrm{Pb}$ data, for the different proton and neutron content of the interacting nuclei, according to the procedure explained in Ref. [3]. The full line is a simple exponential fit of $\exp \left(-\rho_{0} \sigma_{\mathrm{abs}} L\right)$ to the proton and sulphur data, where $\rho_{0}=0.17 \mathrm{fm}^{-3}$ is the nuclear matter average density. It describes the ordinary nuclear absorption of charmonia. It is worthwhile noting that the value obtained for $\sigma_{\text {abs }}$ from this type of simple fit is a good first order approximation of the absorption cross-section of charmonia in nuclear matter when $\sigma_{\mathrm{abs}}$ is small. Strictly speaking, however, the full absorption calculation [14] gives sytematically higher values.

The exponential fit shown in Fig. 6 leads to $5.8 \pm 0.7 \mathrm{mb}$ (equivalent to $6.4 \pm$ $0.8 \mathrm{mb}$ for a full calculation) for the absorption cross-section. The cross-section ratios obtained for the first four $\mathrm{Pb}-\mathrm{Pb}$ bins corresponding to the most peripheral collisions ( $L \leq 8 \mathrm{fm}$ ) can also be described by the same exponential. A clear additional 


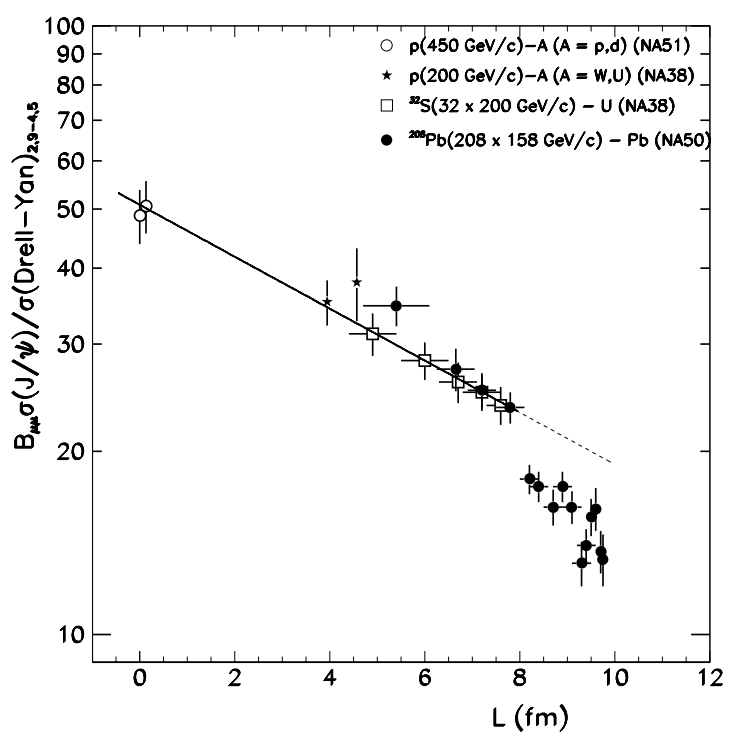

Figure 6: Ratios of the $\mathrm{J} / \psi$ to Drell-Yan cross-sections as a function of the mean nuclear path length $L$, for NA38, NA50 and NA51 data. The NA38 and NA51 data are rescaled to $158 \mathrm{GeV} / c$. The line corresponds to the simple exponential fit with $\sigma_{\mathrm{abs}}=5.8 \mathrm{mb}$.

suppression can be observed for the more central $\mathrm{Pb}-\mathrm{Pb}$ collisions.

\section{The "minimum bias" analysis}

The statistical fluctuations of the ratio $\sigma_{\mathrm{J} / \psi} / \sigma_{\mathrm{DY}}$ are essentially due to the small number of Drell-Yan events. In order to overcome this experimental drawback, an alternative independent analysis has been developed with only the $\mathrm{J} / \psi$ sample of events in common with the standard analysis. The sample of Drell-Yan events is now replaced by the huge sample of minimum bias events and a new estimator of the ratio $\sigma_{\mathrm{J} / \psi} / \sigma_{\mathrm{DY}}$ is built, according to the procedure detailed hereafter.

\subsection{Data selection}

Minimum bias events are those defined by an incident $\mathrm{Pb}$ ion which interacts in the $\mathrm{Pb}$ target of the experiment. They are collected with a beam trigger made of specific hardware which is completely independent of the usual muon pair trigger used for dimuon on-line selection. The beam trigger fires when hadronic energy is measured in the zero degree calorimeter. The minimum detectable zero degree energy does not induce any bias on the $E_{\mathrm{T}}$ distribution since even for head-on collisions a few pions are produced in the acceptance of the ZDC. Although collected simultaneously with the dimuon events, the minimum bias events are prescaled by a factor which brings 
their rate down to about $10 \%$ of the total number of events recorded on tape. In the off-line analysis, a further cut requires $E_{\mathrm{T}}>5 \mathrm{GeV}$.

A key feature of the analysis based on minimum bias events is that, due to the large size of the data samples involved, the target algorithm can be used for sub-target identification despite the induced losses. The result is a constant mass resolution in the whole range from peripheral to central collisions. Furthermore, no fitting procedure is used. The numbers of events are obtained from a simple counting technique. In each $E_{\mathrm{T}}$ bin are counted, independently, the number of minimum bias events and the number of muon pairs in the mass range between 2.9 and $3.3 \mathrm{GeV} / c^{2}$ (after combinatorial background subtraction) which, corrected for the continuum contribution, leads to the number of $\mathrm{J} / \psi$ events.

\subsection{Analysis}

The analysis uses the very similar structure of the transverse energy distributions for minimum bias (MB) and Drell-Yan events (DY) in A-B collisions,

$$
\begin{gathered}
\left(\mathrm{d} N / \mathrm{d} E_{\mathrm{T}}\right)_{\mathrm{MB}}^{\mathrm{th}} \propto \int P\left(E_{\mathrm{T}}, b\right) b \mathrm{~d} b \\
\left(\mathrm{~d} N / \mathrm{d} E_{\mathrm{T}}\right)_{\mathrm{DY}}^{\mathrm{th}} \propto \int N_{A B}(b) P\left(E_{\mathrm{T}}, b\right) b \mathrm{~d} b
\end{gathered}
$$

where $P\left(E_{\mathrm{T}}, b\right)$ is the probability that a given $E_{\mathrm{T}}$ is measured for a collision with impact parameter $b$. The analytical expression of $P\left(E_{\mathrm{T}}, b\right)$ depends on 2 parameters, the mean number of hadrons produced per wounded nucleon in the collision, $\langle h\rangle$, and the mean transverse energy of each of these hadrons, $\left\langle\epsilon_{t}\right\rangle$. The parameters are obtained from a fit to the experimental $E_{\mathrm{T}}$ distributions. Further details on these analytical descriptions can be found in Refs. $[14,15]$ and references therein. $N_{A B}(b)$ is the number of binary nucleon-nucleon collisions, calculated by the Glauber model with the nuclear density described by a three parameter Woods-Saxon distribution. Based on these relations, the Drell-Yan distribution (noted hereafter as $\left(\mathrm{d} N / \mathrm{d} E_{\mathrm{T}}\right)_{\mathrm{DY}} *$ ) can be obtained directly from the minimum bias experimental distribution according to:

$$
\left(\mathrm{d} N / \mathrm{d} E_{\mathrm{T}}\right)_{\mathrm{DY}}=\left(\mathrm{d} N / \mathrm{d} E_{\mathrm{T}}\right)_{\mathrm{MB}}^{\exp } \Theta\left(E_{\mathrm{T}}\right)
$$

with

$$
\Theta\left(E_{\mathrm{T}}\right) \propto\left(\mathrm{d} N / \mathrm{d} E_{\mathrm{T}}\right)_{\mathrm{DY}}^{\mathrm{th}} /\left(\mathrm{d} N / \mathrm{d} E_{\mathrm{T}}\right)_{\mathrm{MB}}^{\mathrm{th}}
$$

The ratio between the theoretical $E_{\mathrm{T}}$ distributions of Drell-Yan and minimum bias events, $\Theta\left(E_{\mathrm{T}}\right)$, is plotted as a function of $E_{\mathrm{T}}$ in Fig. 7. For comparison purposes, the same figure also shows the ratio of the experimental distributions and the number of nucleon-nucleon collisions calculated with the Glauber model, using the Woods-Saxon nuclear density distribution. The agreement of the ratio $\Theta\left(E_{\mathrm{T}}\right)$ with the number of nucleon-nucleon collisions is remarkable all over the range below the knee of the $E_{\mathrm{T}}$ distribution. 


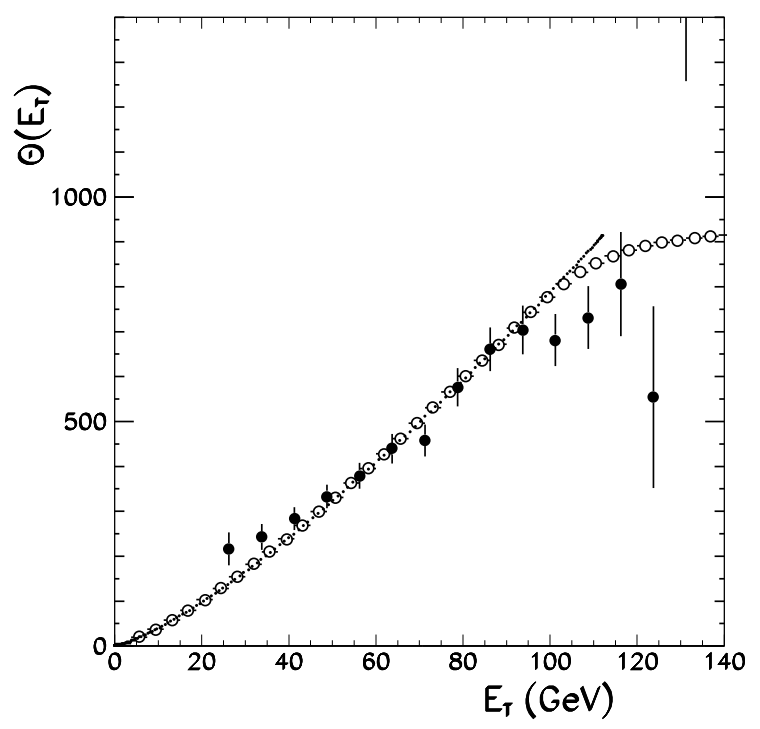

Figure 7: The ratio $\Theta\left(E_{\mathrm{T}}\right)$ of Drell-Yan to minimum bias theoretical $E_{\mathrm{T}}$ distributions (open circles). Also shown is the corresponding ratio for the data (closed circles) and the calculated number of elementary nucleon-nucleon collisions (dotted line).

The cross-sections ratio $\sigma_{\mathrm{J} / \psi} / \sigma_{\mathrm{DY}}$, based on the directly counted $\mathrm{J} / \psi$ and minimum bias events, can be compared to the usual cross-section ratio $\sigma_{\mathrm{J} / \psi} / \sigma_{\mathrm{DY}}$, based on the fitted ratio of $\mathrm{J} / \psi$ and Drell-Yan events, after adjustment on the data of a free normalization constant. The method has a twofold advantage. The cross-section ratio $\sigma_{\mathrm{J} / \psi} / \sigma_{\mathrm{DY}}$ is practically free from statistical fluctuations. It is also free from most inefficiencies as it is still computed from a ratio of experimental numbers. The drawbacks of using minimum bias events are due to potential new systematic effects induced both by their specific trigger (no muons required in the spectrometer) and by their selection criteria (no dimuon-based geometrical cuts) which are different from those used for $\mathrm{J} / \psi$ events.

\subsection{Results}

The ratio of cross-sections $\sigma_{\mathrm{J} / \psi} / \sigma_{\mathrm{DY}} *$ is given in Table 2 for the different $E_{\mathrm{T}}$ bins and plotted in Fig. 8, superimposed on the values obtained with the standard method. The absolute normalization for $\sigma_{\mathrm{J} / \psi} / \sigma_{\mathrm{DY}} *$ was computed in the $E_{\mathrm{T}}$ interval 55$75 \mathrm{GeV}$. The analysis is limited to values above $E_{\mathrm{T}}=26 \mathrm{GeV}$ due to a contamination of off-target interactions which affects, in particular, the more peripheral minimum bias events. The statistical errors are significantly reduced and the shapes obtained by both analyses are compatible. In particular, an excellent agreement is observed between the two sets of points in the energy range $30<E_{\mathrm{T}}<55 \mathrm{GeV}$, where a clear drop of the $\mathrm{J} / \psi$ yield is observed. 
Table 2: Cross-section ratios for the $1996 \mathrm{~Pb}-\mathrm{Pb}$ data as a function of the average neutral transverse energy $\left\langle E_{\mathrm{T}}\right\rangle$. The ratios are obtained from the minimum bias sample of events.

\begin{tabular}{cccc}
\hline $\begin{array}{c}\left\langle E_{\mathrm{T}}\right\rangle \\
(\mathrm{GeV})\end{array}$ & $B_{\mu \mu} \sigma_{\mathrm{J} / \psi} / \sigma_{\mathrm{DY}}$ & $\begin{array}{c}\left\langle E_{\mathrm{T}}\right\rangle \\
(\mathrm{GeV})\end{array}$ & $B_{\mu \mu} \sigma_{\mathrm{J} / \psi} / \sigma_{\mathrm{DY}}$ \\
\hline 28.1 & $28.1 \pm 1.3$ & 76.8 & $16.0 \pm 0.3$ \\
31.8 & $27.2 \pm 0.9$ & 80.6 & $15.2 \pm 0.3$ \\
35.6 & $24.9 \pm 0.6$ & 84.3 & $15.2 \pm 0.3$ \\
39.3 & $23.6 \pm 0.5$ & 88.1 & $14.7 \pm 0.3$ \\
43.1 & $21.7 \pm 0.4$ & 91.8 & $14.3 \pm 0.3$ \\
46.8 & $20.0 \pm 0.3$ & 95.6 & $13.9 \pm 0.2$ \\
50.6 & $19.1 \pm 0.3$ & 99.3 & $13.8 \pm 0.3$ \\
54.3 & $18.8 \pm 0.3$ & 103.1 & $13.0 \pm 0.3$ \\
58.1 & $18.2 \pm 0.3$ & 106.8 & $13.3 \pm 0.3$ \\
61.8 & $17.5 \pm 0.3$ & 110.6 & $13.4 \pm 0.3$ \\
65.6 & $16.7 \pm 0.3$ & 114.3 & $13.6 \pm 0.4$ \\
69.3 & $17.0 \pm 0.3$ & 118.1 & $13.6 \pm 0.5$ \\
73.1 & $16.1 \pm 0.3$ & 121.8 & $13.3 \pm 0.7$ \\
\hline
\end{tabular}

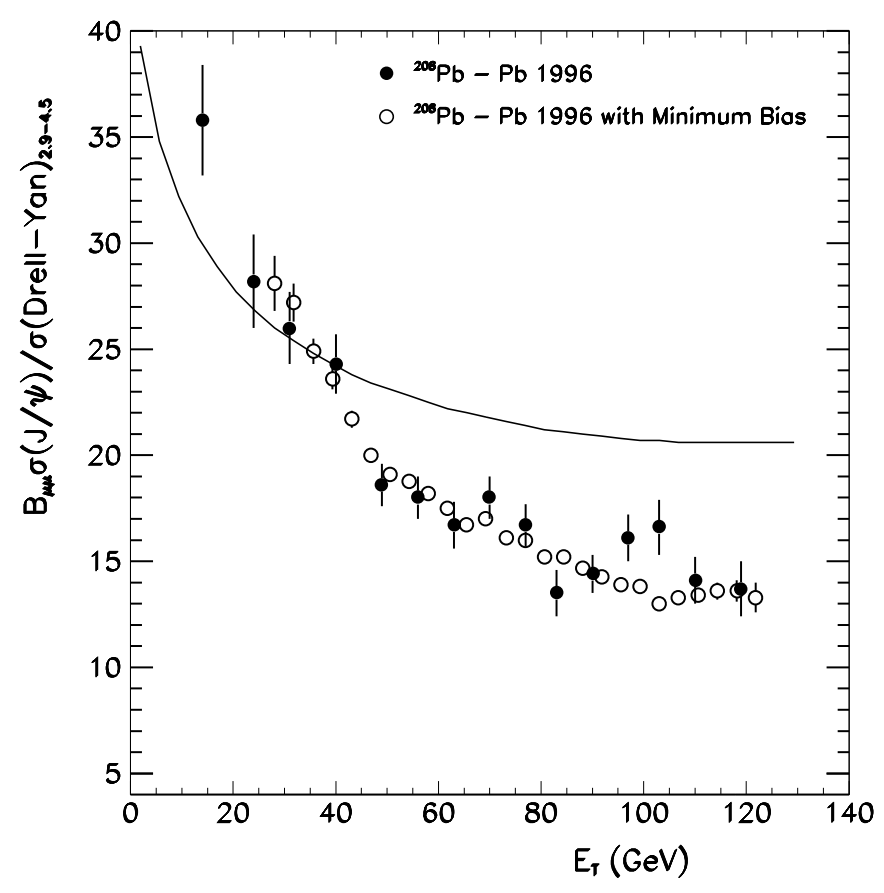

Figure 8: Comparison of the ratios $\sigma_{\mathrm{J} / \psi} / \sigma_{\mathrm{DY}}$ and $\sigma_{\mathrm{J} / \psi} / \sigma_{\mathrm{DY}} *$ based respectively on Drell-Yan and minimum bias events. The curve shows the same ratio for normal nuclear absorption, as plotted in Fig. 5. 


\section{$5 \quad$ Summary and conclusions}

A high statistics data sample of $\mathrm{Pb}-\mathrm{Pb}$ interactions at $158 \mathrm{GeV} / c$ per nucleon incident momentum has been collected in 1996 by experiment NA50. The ratio $\sigma_{\mathrm{J} / \psi} / \sigma_{\mathrm{DY}}$ has been measured as a function of the centrality of the reaction estimated, for each event, from the measured neutral transverse energy $E_{\mathrm{T}}$. The new data have been analyzed with the same method as used for the 1995 smaller sample, except for a new selection procedure which allows to include more peripheral interactions.

The results obtained are in good agreement with the published ones [3, 4], obtained from the data collected in 1995, except at the upper end of the $E_{\mathrm{T}}$ range where there is a difference probably due to re-interactions in the target. These results are corroborated by a new and independent analysis which makes use of the sample of minimum bias events instead of the Drell-Yan events and which, although potentially sensitive to experimental systematic effects, leads to smaller statistical uncertainties. They confirm quantitatively the anomalous $\mathrm{J} / \psi$ suppression previously reported and describe its $E_{\mathrm{T}}$ dependence which much improved accuracy.

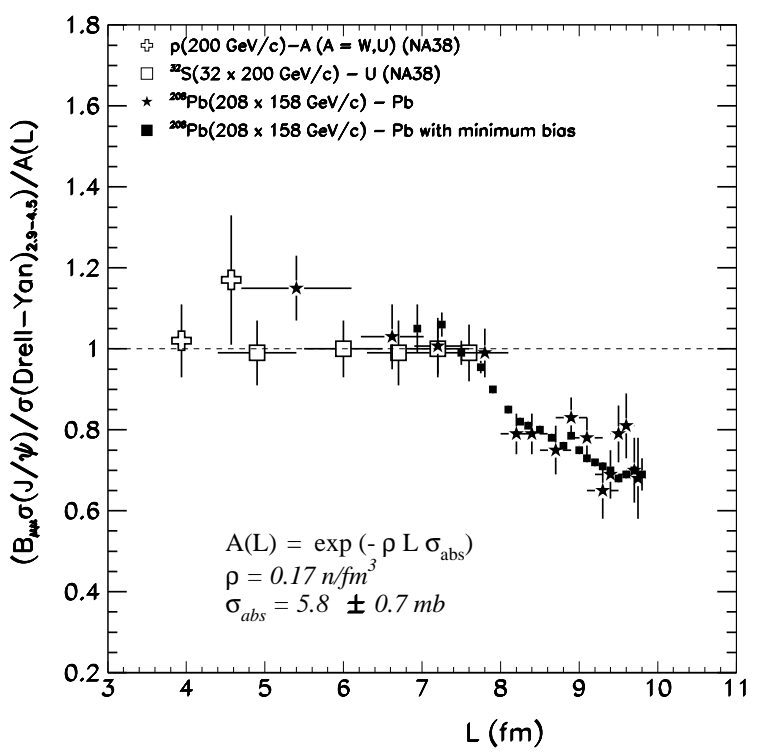

Figure 9: The $\sigma_{\mathrm{J} / \psi} / \sigma_{\mathrm{DY}}$ ratios divided by the normal nuclear absorption suppression inferred from the NA38 and NA51 results.

The large size of the 1996 data sample allows a detailed study of the anomaly as a function of centrality. Whereas peripheral events exhibit the normal behaviour already measured for lighter projectiles or targets, the $\mathrm{J} / \psi$ shows a significant anomalous drop of about $20 \%$ in the $E_{\mathrm{T}}$ range between 40 and $50 \mathrm{GeV}$, which approximately corresponds to an impact parameter between 7.3 and $8.3 \mathrm{fm}$. The drop can only be due to the $\mathrm{J} / \psi$ sample since it is the only sample of events in common in the two methods. The detailed pattern of the anomaly can be seen in Fig. 9 which shows the ratio of the $\mathrm{J} / \psi$ to the Drell-Yan cross-sections divided by the exponentially decreasing function accounting for normal nuclear absorption. 
Charmonium suppression is expected to sign the phase transition between normal and deconfined nuclear matter predicted by lattice QCD calculations. The sudden change of behaviour observed in our data suggests that the observed abnormal suppression results from a discontinuity in the state of nuclear matter. The normal $\mathrm{J} / \psi$ cross-section measured for peripheral $\mathrm{Pb}-\mathrm{Pb}$ collisions agrees with the general pattern determined from proton, oxygen and sulphur-induced reactions which can be accounted for by nuclear absorption. A clear onset of the anomaly is observed as a function of transverse energy. It excludes models based on hadronic scenarios since only smooth behaviours with monotonic derivatives can be inferred from such calculations.

We would like to acknowledge the constant and efficient support of CERN and, in particular, the fundamental contributions of the PS and SL groups which have provided a $\mathrm{Pb}$ beam of good stability, high intensity and low emittance as required by the experiment. The fine tuning and focussing of the halo-free final beam have created the conditions which allowed to develop and use the new methods described in this paper. We are indebted to J. Gosset, from SPhN, Saclay, for pertinent remarks on our 1995 results and deep interest on the data analysis. This work was partially supported by Fundação para a Ciência e a Tecnologia and by INTAS grant 96-0231. One of the authors (L.C.) acknowledges financial support from the EC (TMR programme) under contract number ERBFMBICT961204.

\section{References}

[1] T. Matsui and H. Satz, Phys. Lett. B 178 (1986) 416.

[2] M.C. Abreu et al. (NA51 Coll.), Phys. Lett. B 438 (1998) 35.

M.C. Abreu et al. (NA38 Coll.), Phys. Lett. B 444 (1998) 516;

M.C. Abreu et al. (NA38 Coll.), accepted by Phys. Lett. B (CERN-EP/98-190).

[3] M.C. Abreu et al. (NA50 Coll.), Phys. Lett. B 410 (1997) 337.

[4] M.C. Abreu et al. (NA50 Coll.), Phys. Lett. B 410 (1997) 327.

[5] R. Arnaldi et al., Nucl. Instr. Meth. A 411 (1998) 1.

[6] M. Gonin et al. (NA50 Coll.), Proceedings of the RHIC'97 Summer Study, Brookhaven, July 1997;

L. Ramello et al. (NA50 Coll.), Nucl. Phys. A 638 (1998) 261c.

[7] H. Sorge et al., Phys. Lett. B 243 (1990) 7; Phys. Lett. B 271 (1991) 37.

[8] F. Bellaiche et al., Nucl. Instr. Meth. A 398 (1997) 180.

[9] T. Sjöstrand, Comp. Phys. Comm. 82 (1994) 74.

[10] A.D. Martin et al., Phys. Lett. B 306 (1993) 145; Phys. Lett. B 410 (1997) 337.

[11] R.M. Barnett et al., Phys. Rev. D 54 (1996) 1.

[12] E. Scomparin et al. (NA50 Coll.), Proceedings of the 4th Int. Conf. on Strangeness in Quark Matter, Padova, Italy, July 1998 (CERN-EP/98-188).

[13] C. Gerschel and J. Hüfner, Z. Phys. C 56 (1992) 171.

[14] D. Kharzeev et al., Z. Phys. C 74 (1997) 307.

[15] C. Baglin et al. (NA38 Coll.), Phys. Lett. B 251 (1990) 472. 